MoPpm4-01, Ver: 1.0, 2005.08.26

\title{
Title: Viscoelasticity evaluation of rubber by surface reflection of supersonic wave
}

\section{Author:}

Nobuaki Omata ${ }^{\mathrm{a}}$, Takahiro Suga ${ }^{\mathrm{a}}$, Hirokazu Furusawa ${ }^{\mathrm{a}}$, Shinichi Urabe ${ }^{\mathrm{a}}$ and Takeru Kondo ${ }^{\mathrm{b}}$ and Qing-Qing $\mathrm{Ni}^{\mathrm{c}}$

\section{Address:}

a: OMRON Corporation, Kyoto, JAPAN

b: Kyoto institute of technology, Kyoto, JAPAN

c: Shinshu University, Nagano, JAPAN

\begin{abstract}
The main characteristic of rubber is a viscoelasticity. So it is important to research the characteristic of the viscoelasticity of the high frequency band for the friction between a rubber material and the hard one with roughness, for instance, the tire and the road. As for the measurement of the viscoelasticity of rubber, DMA (Dynamic Mechanical Analysis) is general. However, some problems are pointed out to the measurement of the high frequency band by DMA. Then, we evaluated the viscoelasticity characteristic by the supersonic wave measurement. However, attenuation of rubber is large, and when the viscoelasticity is measured by the supersonic wave therefore, it is inconvenient and limited in a past method by means of bottom reflection. In this report, we tried the viscoelasticity evaluation by the method of using complex surface reflection coefficient and we compared with the friction coefficient under wide range friction velocity. As a result, some relationships had been found for two properties. We report the result that character of viscoelasticity of rubber was comparable to friction coefficient.
\end{abstract}

\section{Key words:}

viscoelasticity, complex surface reflection, friction coefficient, tire, supersonic wave

\section{Contact author: Nobuaki Omata}

OMRON Corporation Industrial Automation Company. Technology Development Center HQ,

9-1, Kizugawadai, Kizu-cho, Soraku-gun, Kyoto, 619-0283 JAPAN

Fax: +81-774-74-2003,

E-mail: nobuaki_omata@omron.co.jp 


\section{Introduction}

It is understood that there is a deep relationship between the friction of the tire and the viscoelasticity, especially loss tangent $\tan \delta$ being researched for years.[1-3] Moreover, it is said that the friction frequency are the high frequency band $\left(10^{2} \sim 10^{6} \mathrm{~Hz}\right)$. However, a general viscoelasticity evaluation method DMA (Dynamic Mechanical Analysis) is limited in the low frequency $\left(10^{1} \sim 10^{2} \mathrm{~Hz}\right.$ )measurement. For the viscoelasticity evaluation at the high frequency band, it was necessary to do the time-temperature conversion that had the problems like a conversion error margin or a temperature increasing time. Therefore, it is not possible to measure in the state of actual use, so that the measurement was limited in the laboratory. For the supersonic, a vibration of the high frequency band, viscoelasticity measurement of the solid polymer, a basic theory and measurement result used the rubber sample in the water tank were reported[4,5]. However, the measuring method for the real shape and actual use rubber commodity had not established. Moreover, the relationship between the supersonic wave viscoelasticity and the friction coefficient was not examined.

Authors compared the friction coefficient of rubber and paper at a wide-rang sliding velocity with tan $\delta$ by DMA and the supersonic wave, and qualitative correlation was reported.[6,7] In these reports, tan $\delta$ was calculated from the attenuation coefficient and the acoustic phase velocity of rubber by the bottom reflection method using the bottom reflection wave propagation through the inside of the rubber sample. However, because of rubber was the well known high attenuation material, there were the problems that the reflection wave signal from the bottom of sample can't obtain enough strength, an enough flatness and reflectivity were necessary for the sample bottom in a actual use.

To solve such problems, we are able to remember the method that complex modulus is calculated by complex reflection coefficient measured by the reflection wave on the surface of the sample. As the viscoelasticity measuring method using surface reflection coefficient, the oblique angle incidence $\mathrm{SH}$ wave method using quartz buffer has been researched since 1940's [8-11]. However measuring object was mostly a liquid and there were few reports about a solid material such as cured rubber.

The SH wave must be chosen in this oblique angle incidence method for reduction the mode transformation error margin. However, in the propagation of the shear wave such as SH wave, it was difficult to request enough reproducibility in the surface reflection method by the phase difference of the reflection wave from the boundary of sample and buffer, because the bonding accuracy of the solid sample and solid buffer have a big influence on the measurement accuracy.

To solve the problem of the surface reflection wave viscoelasticity measurement method, we have evaluated the surface reflection method by a longitudinal wave vertical incidence. We think the mode transformation may be little by means of the vertical incidence, and a longitudinal wave also makes the bonding easier. We hope this method will be able to be applied to the viscoelasticity measurement for actual use shape of rubber product like a tire.

In this paper, we report our study about relationship between viscoelasticity and friction by comparing 
the supersonic viscoelasticity measurement method using longitudinal vertical incidence surface reflection wave from boundary of buffer and sample with the frequency property of friction coefficient of rubber.

\section{Theory and experimental apparatus}

\subsection{Examination material}

The measuring samples that were mixed the silica particle with three varieties of matrix rubbers of butadiene rubber (BR), styrene butadiene rubber (SBR), and polynorbornene rubber (PNR) were prepared. BR and SBR are popular materials for the tire. PNR was popular material for the paper feeding roller. As you can see in Table 1, one of the viscoelastic parameter the glass transition temperature $T_{g}$ measured by DSC(Differential Scanning Calorimetry) for each rubber samples shows a big different value.

\subsection{Surface reflection supersonic wave viscoelasticity measuring method}

The schematic of the supersonic viscoelasticity measuring method composition using the surface reflection wave is shown in Fig.1. Supersonic transducers were the one for NDT(non-destructive-testing), total band of $0.5 \sim 100 \mathrm{MHz}$. The pulsar receiver was also the one for NDT, generate pulse wave form. Wave form $U_{A}$ of surface reflection wave that was obtained through the oscilloscope was analyzed by Fast Fourier Transform with the personal computer. The absolute value of reflection coefficient from the power spectrum and the phase delay $\theta$ from the phase spectrum were calculated standardized based on the reflection with air $U_{A 0}$.

$$
\begin{aligned}
& R=\frac{U_{A}}{U_{A 0}}=\frac{A \mathrm{e}^{i \theta_{A}}}{A_{0} \mathrm{e}^{i \theta_{A 0}}}=|R| \mathrm{e}^{i \theta} \\
& |R|=\frac{|A|}{\left|A_{0}\right|} \\
& \theta=\theta_{A}-\theta_{A_{0}}
\end{aligned}
$$

Complex reflection coefficient $R$ was calculated by equations (1), (2), (3),

Storage modulus $L^{\prime}$, Loss modulus $L^{\prime \prime}$ and Loss tangent $\tan \delta$ was calculated by equations (4), (5), (6).

$$
\begin{aligned}
& L^{\prime}=\frac{Z_{\text {Buffer }}{ }^{2}}{\rho} \frac{\left(1-|R|^{2}\right)^{2}-4|R|^{2} \sin ^{2} \theta}{\left(1+2|R| \cos \theta+|R|^{2}\right)^{2}} \\
& L^{\prime \prime}=\frac{Z_{\text {Buffer }}{ }^{2}}{\rho} \frac{4 \mid R\left(1-|R|^{2}\right) \sin \theta}{\left(1+2|R| \cos \theta+|R|^{2}\right)^{2}} \\
& \tan \delta=\frac{L^{\prime \prime}}{L^{\prime}}=\frac{4|R|\left(1-|R|^{2}\right) \sin \theta}{\left(1-|R|^{2}\right)^{2}-4|R|^{2} \sin ^{2} \theta}
\end{aligned}
$$


Where $Z_{\text {Buffer }}$ is a acoustic impedance of buffer material, and $\rho$ is a density of sample

\subsection{Viscoelasticity measuring method with rheometer}

The schematic of the measuring apparatus for viscoelasticity evaluation (rheometer made by the ANTON PAAR company) to give the dynamic twist strain and to detect the torque is shown in Fig.2. (Above-mentioned DMA is measuring method under a tensile strain)

Sine rotatory vibration $u$ of $0.01 \sim 10 \mathrm{~Hz}$ was given to an end of the specimen, and phase difference $\delta$ between the detected shear stress $\tau_{0}$ and strain were measured within the range from -100 to $25^{\circ} \mathrm{C}$. Then Storage modulus $G^{\prime}$, Loss modulus $G^{\prime \prime}$ and Loss $\operatorname{tangent} \tan \delta$ were obtained by equations (7)to (12). In this experiment, shear strain $\gamma_{0}$ was $0.02 \%$, and specimen thickness $h$ was $1 \mathrm{~mm}$, and specimen length $l$ was $30 \mathrm{~mm}$.

$$
\begin{gathered}
\quad \mathcal{L}=u_{0} \sin \omega t \\
\tau=\tau_{0} \sin (\omega t+\delta) \quad(0<\delta<\pi / 2)
\end{gathered}
$$

where $\omega=2 \pi f$.

$$
\begin{aligned}
& \gamma_{0}=u_{0} / \ell \\
& G^{\prime}=\left(\sigma_{0} / \gamma_{0}\right) \cos \delta \\
& G^{\prime \prime}=\left(\sigma_{0} / \gamma_{0}\right) \sin \delta \\
& \tan \delta=G^{\prime \prime} / G^{\prime}
\end{aligned}
$$

The master curve of the frequency dependency dynamic property at optional temperature $T$ is obtained by moving a measured curve on the time axis. It is called time-temperature conversion shown as following WLF equation here $\log a_{T}$ that is expressed by the amount of the movement along logarithm axes of the frequency. [12]

WLF equation

$$
\begin{aligned}
& \log _{10} \alpha_{T}=-\frac{8.86\left(T-T_{s}\right)}{101.6+T-T_{s}} \\
& T_{s}=T_{g}+50 \mathrm{~K}
\end{aligned}
$$

At first the measurement result had been converted into the frequency according to the WLF equation based on glass transition temperature $T_{g}$, next it was shifted to $23^{\circ} \mathrm{C}$.

\subsection{Friction coefficient measuring method}

The rubber roller friction examination apparatus that authors developed for the evaluation of the bank note handling is shown in fig.3. With this apparatus, wide range velocity $(0.01 \sim 3.0 \mathrm{~m} / \mathrm{s})$ friction coefficient measurmente was realized, (a) was set up for ultra low velocity and (b) was for high velocity. The friction coefficient was calculated by equation (15). Friction force $F$ was measured by load-cell.

$$
\mu=F / N
$$


The pressing load $N$ was $3.6 \mathrm{~N}$. The friction vibration frequency $f$ was calculated from the friction velocity and the surface roughness of paper.

$$
f=V / \lambda \times 10^{-6} \quad \mathrm{~Hz}
$$

where $V$ is relative velocity of paper and rubber roller $(\mathrm{m} / \mathrm{s})$, and $\lambda$ is average wave length of surface roughness of paper $(\lambda=5 \mu \mathrm{m})$.

From equation (16), the sliding vibration frequency by surface roughness of paper is $2 \times 10^{2} \sim 5.6 \times 10^{5} \mathrm{~Hz}$ at measuring friction velocity $V=0.01 \sim 2.8 \mathrm{~m} / \mathrm{s}$.

\section{Experimental result and consideration}

The frequency property of a viscoelasticity $\tan \delta$ with the supersonic surface reflection method and the rheometer and friction coefficient $\mu$ of three kinds of the tire and roller material are shown in Fig.4.

The friction coefficient $\mu$ is $10^{3} \mathrm{~Hz}$ shifted as the peak align to $\tan \delta$ in (b)[3].

In BR that glass transition temperature is low, $\tan \delta$ increases by an increase of the frequency. And in PNR that glass transition temperature is high, $\tan \delta$ decreases by an increase of the frequency. And in SBR that glass transition temperature is middle of other two material, $\tan \delta$ shows the peak in $10^{7} \mathrm{~Hz}$. That is to say, $\tan \delta$ with the supersonic surface reflection method and the rheometer shows a similar tendency in three kinds of materials. However, It is difficult to compare rheometer with supersonic tan $\delta$ absolutely. Because restricting condition, stress type and magnitude of vibration were different in each method. Furthermore the measurement with rheometer which is necessary for time-temperature conversion is thought involving the big error margin of the frequency according to how to take the glass transition temperature or coefficients.

On the other hand, as for frequency property of friction coefficient, friction coefficient $\mu$ of BR increases by an increase of the frequency. SBR shows the peak, then, BR and SBR show similarity in viscoelasticity property in the inclination, the amplitude or the peak frequency. As for PNR friction coefficient $\mu$ behavior was different from the tendency to $\tan \delta$, because the friction coefficient did not show a definite change to frequency increase and it was very small.

As for the measurement in $100 \mathrm{MHz}$, now it became to be able to be detected by the new surface reflection method, it had been impossible to be detected in a past bottom reflection method by great attenuation of rubber. The profile of the frequency property of $\tan \delta$ became clear so that the comparison of tendencies became easier.

Fig.5 shows a relationship between friction coefficient $\mu$ and $\tan \delta$.

In the area that value of $\tan \delta$ is 0 , it is assumed that the main factor of the friction force is the adhesive friction. It is said that this value will be decided depending on different shear strength according to the material and a contact area [13]. If we thought in this way, the adhesive friction coefficient of each material are 1.4 in BR, 2.5 in SBR and 0.7 in PNR. Not only shear strength of rubber, 
the adhesive force of paper and rubber but also the energy loss of wear-out by shear strength of paper is included in this adhesive friction coefficient. It is considered to make up to pretty big weight in the friction of rubber. We suspect it's complication

Moreover, as for BR and SBR, the value that subtracts the above-mentioned adhesion friction from total coefficient of friction was in proportion to $\tan \delta$, however, PNR is almost unrelated. Therefore, the influence level to friction coefficient $\mu$ to $\tan \delta$ is different in each material.

From these discussions, the viscoelasticity property by the supersonic measurement using the surface reflection has good correlations for the viscoelasticity with the rheometer. Moreover, since it correlates to the friction coefficient well of materials, it can be said that there is a possibility of evaluation for change like a deterioration or a presumption for a friction coefficient by means of supersonic measurement.

\section{Conclusion}

We introduced the theory and the measurement result of a practical viscoelasticity measurement using the surface reflection wave about the material for the tire and rubber roller that was the high attenuation rubber material. At the result of comparison friction coefficient in wide-range velocity with the supersonic viscoelasticity $\tan \delta$, these showed good correlation in the material used for the tire.

We think that the supersonic viscoelasticity evaluation method applicated to the friction evaluation had reached to a practical level. Because the supersonic viscoelasticity measuring method in an actual friction frequency on the surface for a real friction face was established, so that the viscoelasticity characteristic on the surface that influences friction directly can be understood.

It is convinced that the enormous progress can be given to the safety of the car for an application of this research, because it is possible to realize more safety tire by developing the good friction material or to improve the vehicle inspection quality in the actual use scene by to be able to detect the viscoelasticity deterioration.

\section{References}

[1] K.A.Grosch: Rubber Chemistry and Technology, 37(1964) 386

[2] K.C.Ludema, D.Tabor: Wear, 9 (1966) 329

[3] D.F.Moore: The Friction and Lubrication of Elastmers, Pergamon Press, oxford (1972) 23

[4] Junichi Saneyoshi, supersonic wave technological handbook, Daily Industrial Newspaper Co. (1978) 606

[5] M.Grespacher: High Frequency Viscoelasticity of Carbon Black Filled Compounds, Rubber Chem.Technol. , 69(1996) 786.

[6] Nobuaki Omata, Akimasa Sasaki, Qin-Qin Ni: High Frequency Dynamic Viscoelasticity Measuring Method for Frictional Characteristics Evaluation of Rubber Roller for Paper Handling (Part I), 
Tribologist, 50- 2 (2005) 142.

[7] Nobuaki Omata, Qin-Qin Ni, Yuki Miyamoto, Masaharu Iwamoto: High Frequency Dynamic Viscoelasticity Measuring Method for Frictional Characteristics Evaluation of Rubber Roller for Paper Handling (Part II), Tribologist, 50- 3 (2005) 238.

[8] H.T.O'Neil, Physical Review, 75 (1949) 928

[9] W.P.Mason, W.O.Baker, H.J.McSkimin and J.H.Heiss, Physical Review, 75 (1949) 936

[10] S.M.Crawford, Proc.Phys.Soc, 69B (1956) 1312

[11] I.Alig, S.Tadjbakhsch and A.Zosel: Comparison of ultrasonic shear waves and dynamic-mechanical measurements in acrylic-type copolymers, J. Polymer Sci. PartB: Polymer Phys., 36 (1998) 1703.

[12] J.D.Ferry: Viscoelastic propertys of polymers 3rd, John Wiley \& Sons inc, (1980) 289.

[13] Naoya.Amino, Yoshitaka.Uchiyama, Tomoaki.Iwai: Studies on the Friction Mechanism of Silica-Filled and Carbon Black-Filled SBRs,Part 2.Friction on a Wet Surface, The Society of Rubber Industry, Japan magazine, 74, 3 (2001) 110. 
Table $1 \quad$ Examination material

\begin{tabular}{|c|c|c|c|}
\hline Group & $\begin{array}{c}\text { Hardness } \\
\text { (JIS-A) }\end{array}$ & Filler* & $T_{g}, \quad{ }^{\circ} \mathrm{C}$ \\
\hline B R & 40 & $\mathrm{Si}$ & -105 \\
\hline SBR & 40 & $\mathrm{Si}$ & -50 \\
\hline PNR & 40 & $\mathrm{CB}$ & -4 \\
\hline
\end{tabular}

* Si : Silica, CB : Carbon Black 
Fig. 1 Schematic of measurement apparatus for supersonic viscoelasticity evaluation by surface reflection

Fig. 2 Schematic of measurement apparatus for viscoelasticity evaluation by rheometer

Fig. 3 Schematic of measurement apparatus for friction coefficient of rubber roller and paper

Fig. 4 Frequency relationship between supersonic $\tan \delta$ and rheometer $\tan \delta$ and friction coefficient $\mu$

Fig. 5 Relationship between supersonic $\tan \delta$ and friction coefficient $\mu$ 
Fig.1, NOBUAKI OMATA, MoPpm4-01

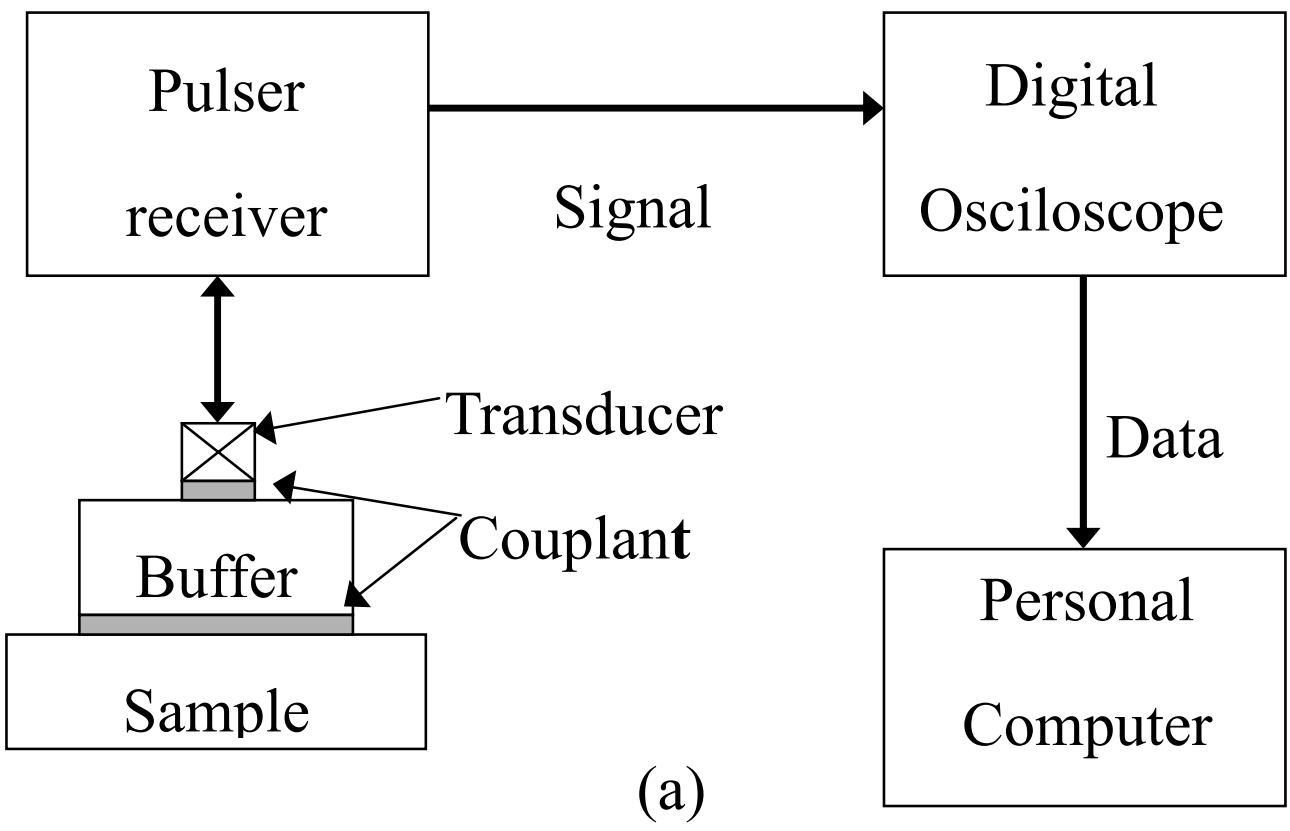

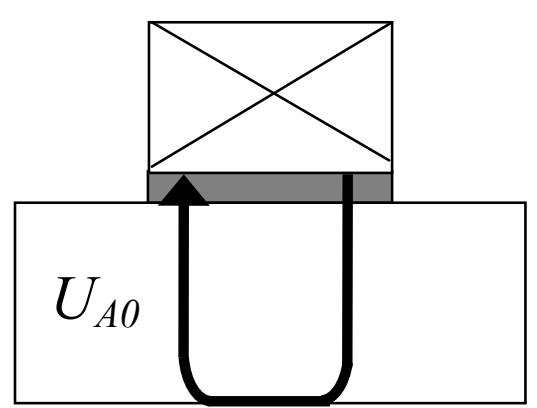

(b)

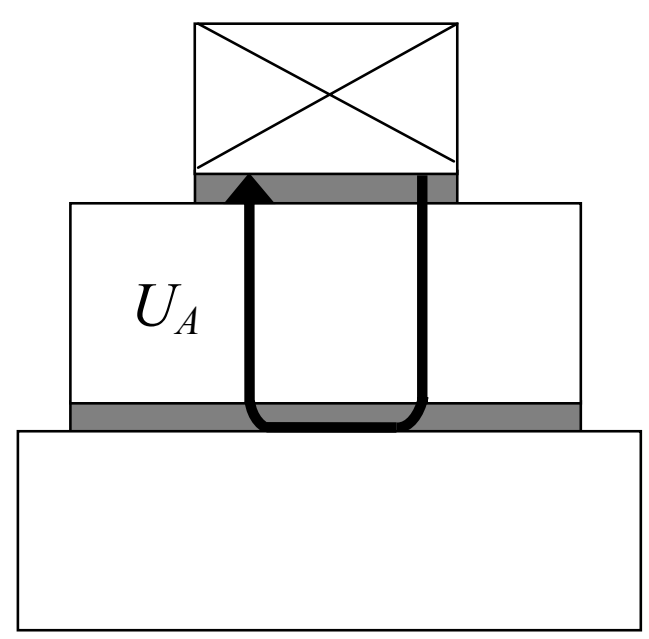

(c) 
Fig.2, NOBUAKI OMATA, MoPpm4-01

Stress sensor

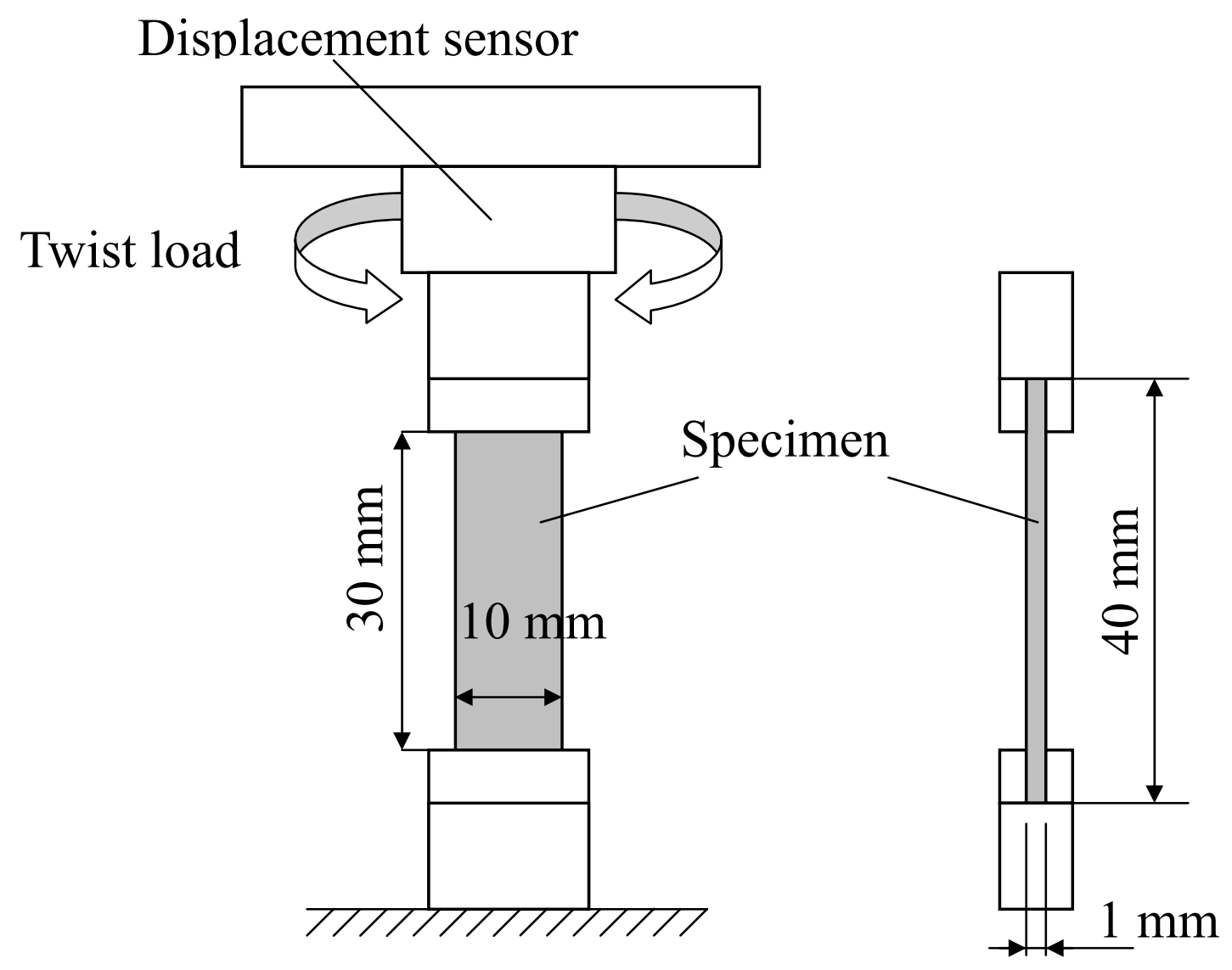


Fig.3, NOBUAKI OMATA, MoPpm4-01

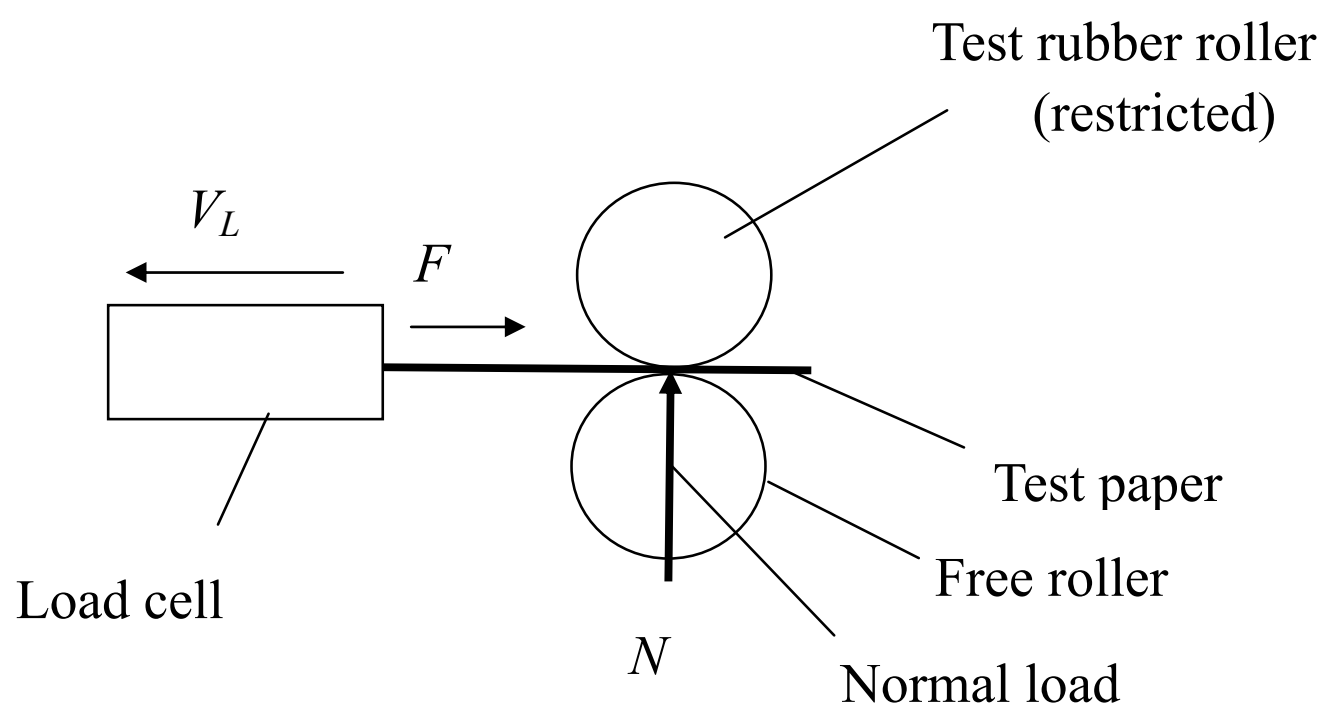

(a) Restricted rubber roller

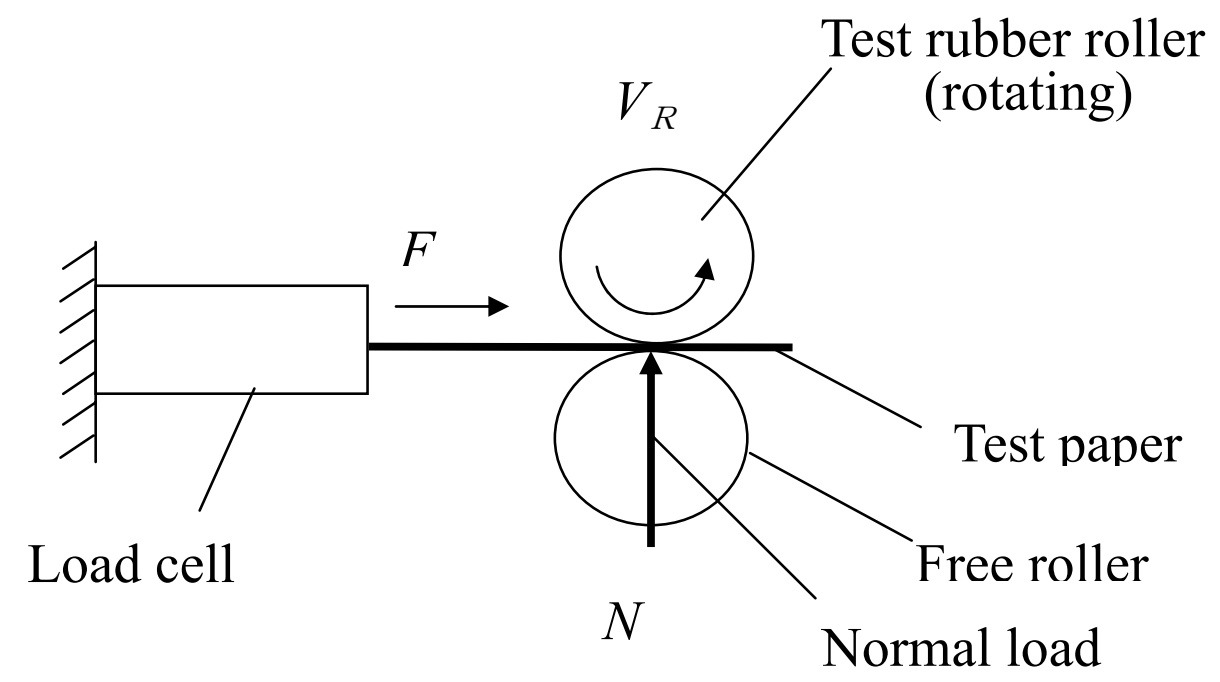

(b) Rotating rubber roller 
Fig.4-a, NOBUAKI OMATA, MoPpm4-01

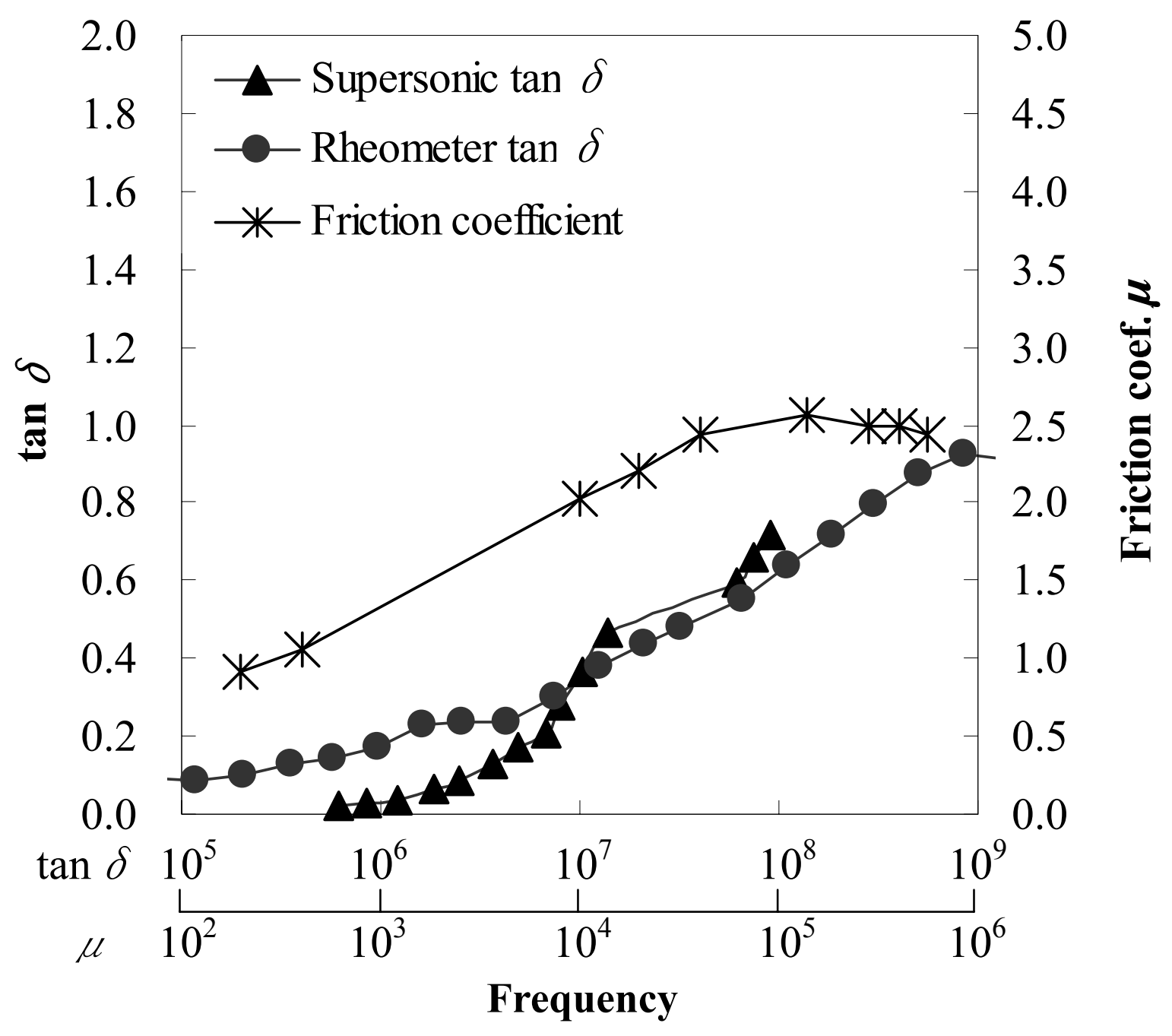

(a) BR 
Fig.4-b, NOBUAKI OMATA, MoPpm4-01

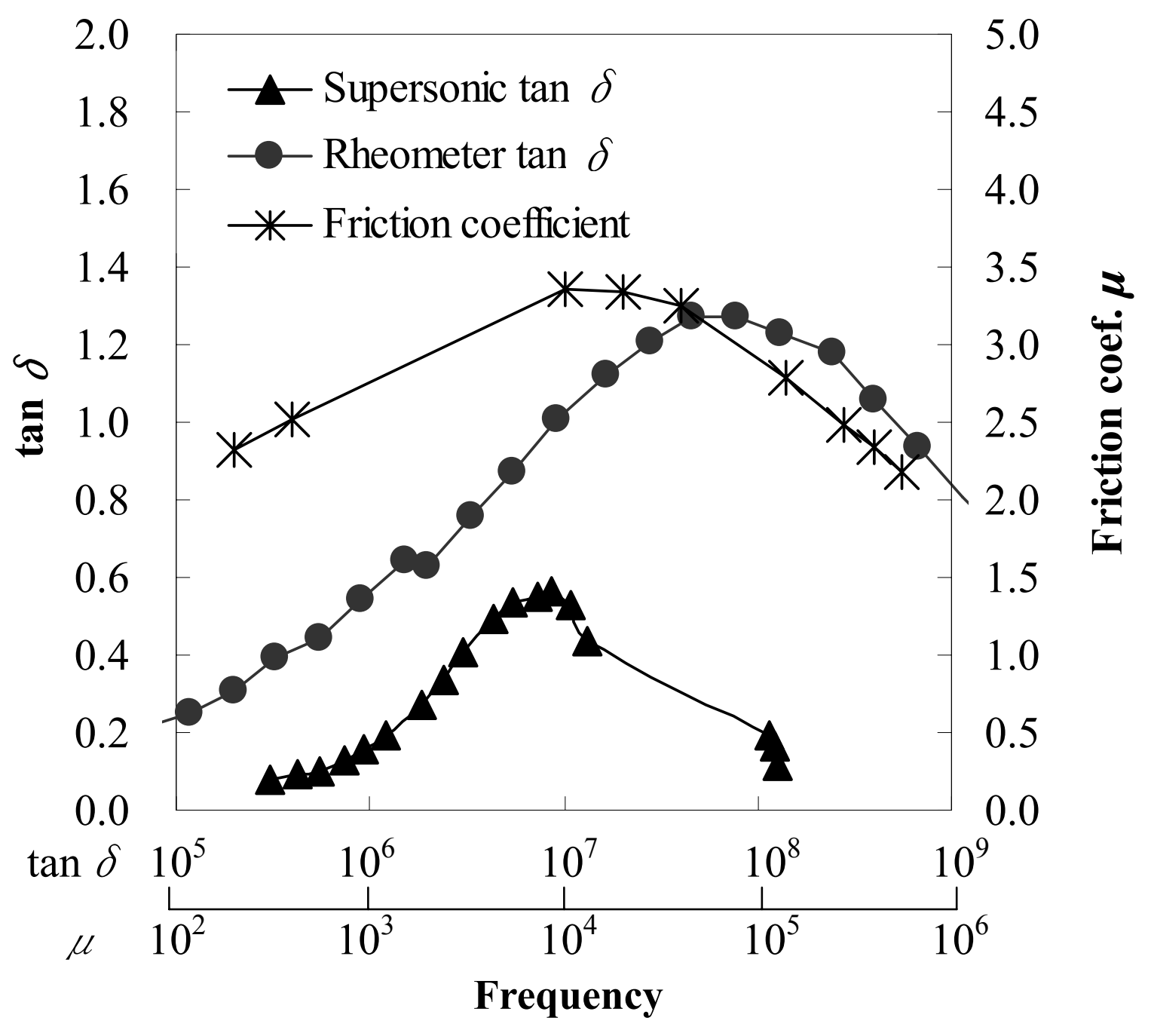

(b) SBR 
Fig.4-c, NOBUAKI OMATA, MoPpm4-01

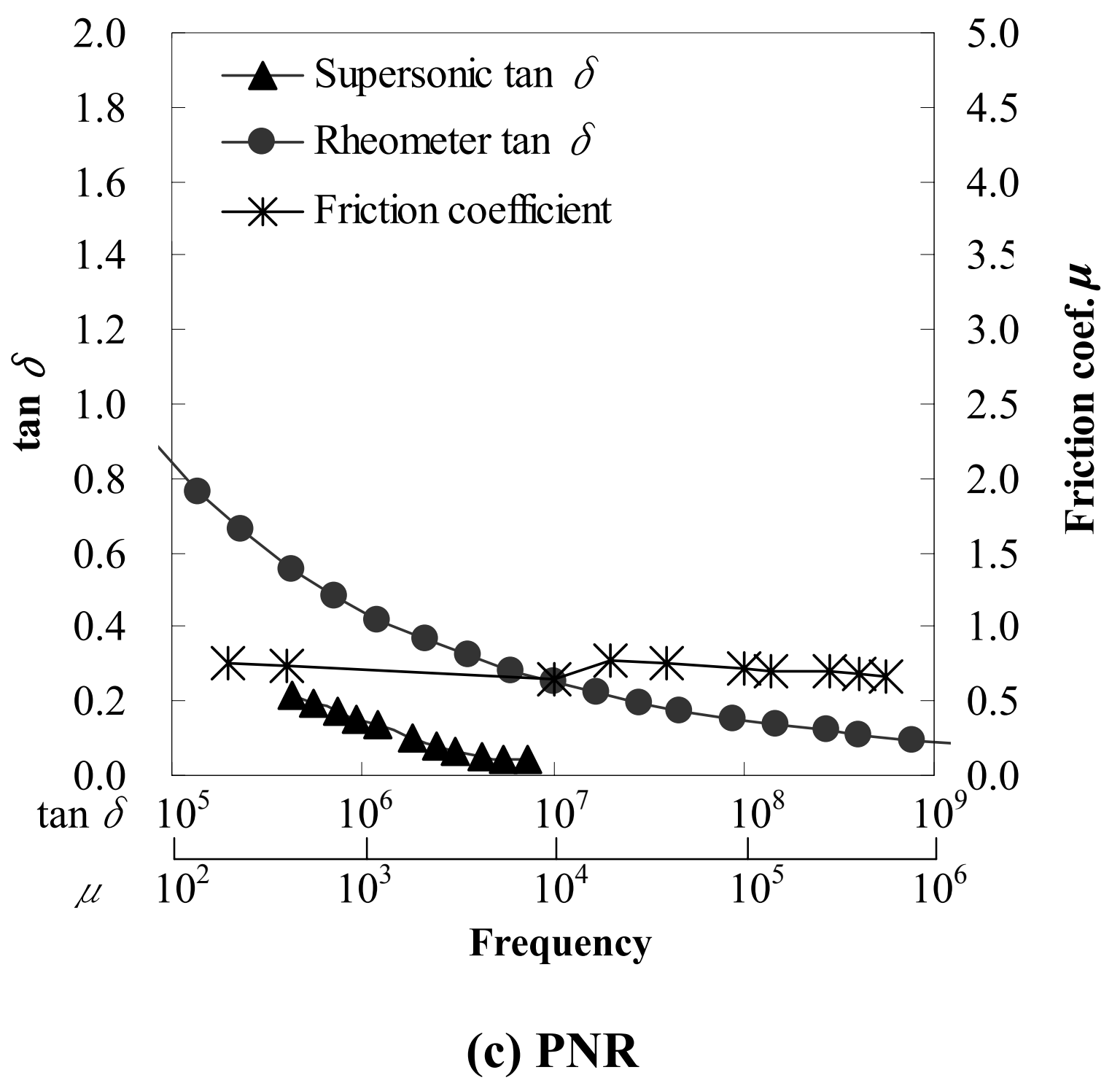


Fig.5, NOBUAKI OMATA, MoPpm4-01

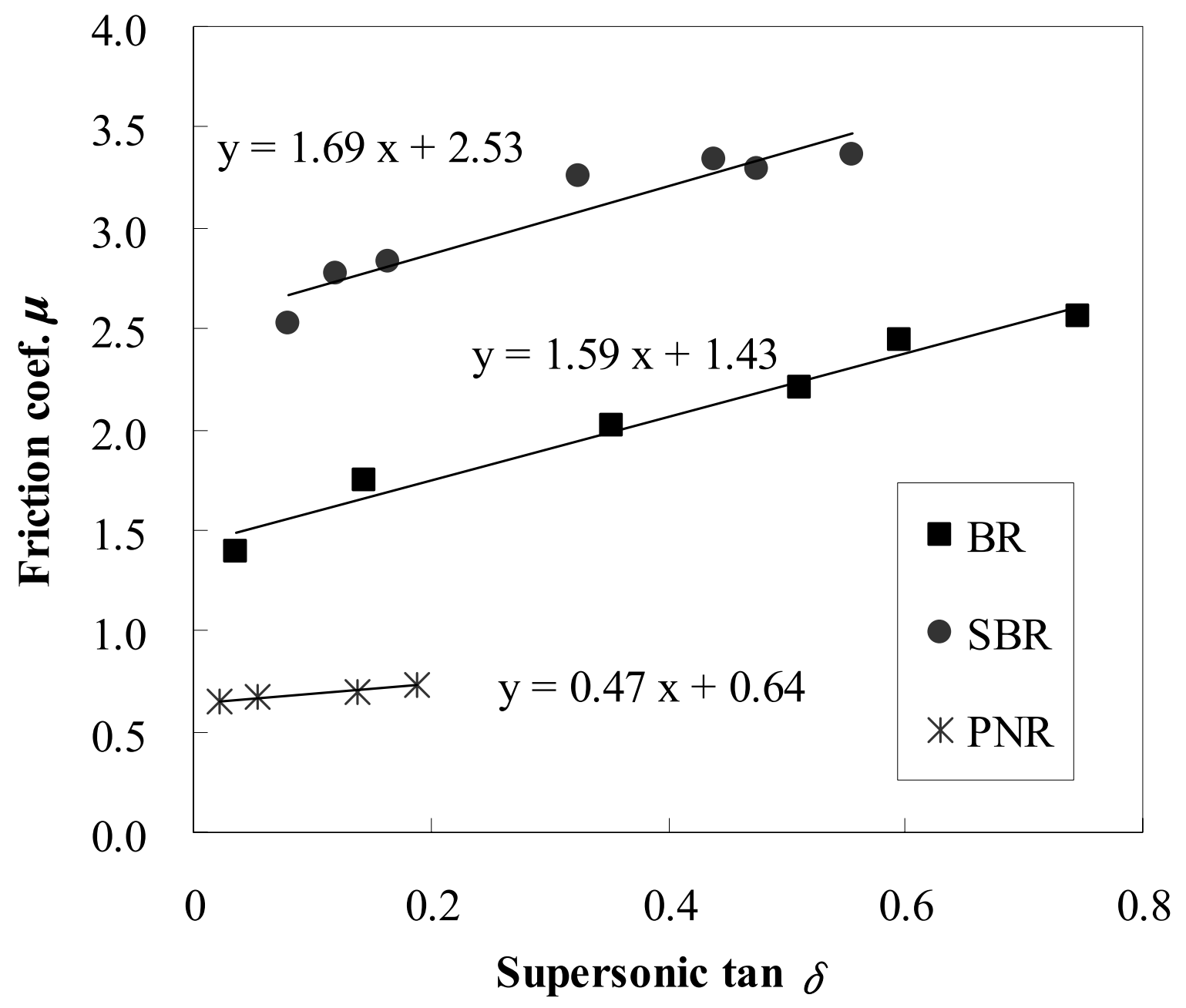

